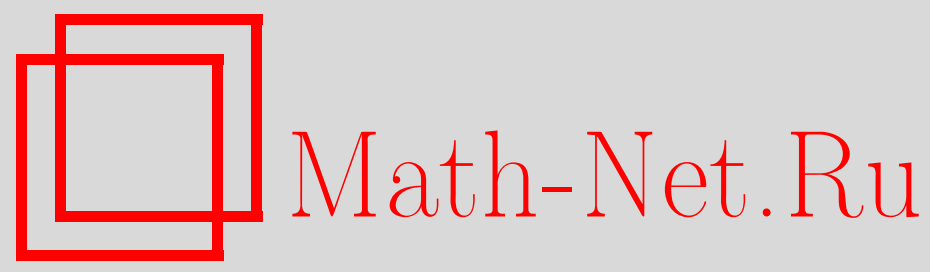

А. С. Белов, Об одном условии сходимости в среднем тригонометрических рядов, Матем. заметки, 2001, том 69, выпуск 3, 323-328

DOI: https://doi.org/10.4213/mzm505

Использование Общероссийского математического портала Math-Net.Ru подразумевает, что вы прочитали и согласны с пользовательским соглашением http://www.mathnet.ru/rus/agreement

Параметры загрузки:

IP: 54.224 .187 .69

26 апреля 2023 г., $14: 15: 10$

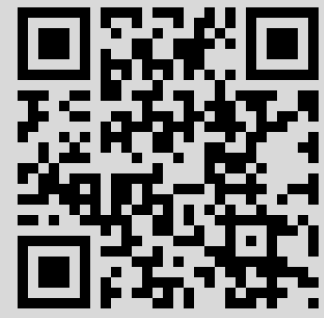


УДК 517.5

\title{
ОБ ОДНОМ УСЛОВИИ СХОДИМОСТИ В СРЕДНЕМ ТРИГОНОМЕТРИЧЕСКИХ РЯДОВ
}

\author{
А. С. Белов
}

В статье строится пример тригонометрического ряда, который показывает неулучшаемость одного условия, тесно связанного со сходимостью в среднем тригонометрических рядов.

Библиографйл: 6 названий.

Пусть задан произвольньй тригонометрический ряд

$$
\sum_{n=-\infty}^{\infty} c_{n} e^{i n x}
$$

Как обычно,

$$
\begin{gathered}
S_{n}(x)=\sum_{k=-n}^{n} c_{k} e^{i k x}, \quad \sigma_{n}(x)=\frac{1}{n+1} \sum_{k=0}^{n} S_{k}(x) \\
\widetilde{S}_{n}(x)=\sum_{k=1}^{n} i\left(-c_{k} e^{i k x}+c_{-k} e^{-i k x}\right)
\end{gathered}
$$

при всех $n \geqslant 0$. Если $f \in L_{2 \pi}$, то

$$
\|f\|=\|f\|_{1}=\|f\|_{L}=\frac{1}{2 \pi} \int_{0}^{2 \pi}|f(x)| d x .
$$

Известно (см. [1, гл. $1, \S 60])$, что если ряд (1) является рядом Фурье, то его частные суммы $S_{n}$ сходятся (ограничены) в метрике $L$ тогда и только тогда, когда

$$
\left\|\sigma_{n}-S_{n}\right\|=o(1) \quad(\text { соответственно }=O(1)) .
$$

В (2) и далее в аналогичных соотношениях предполагаем, конечно, что $n$ стремится к бесконечности.

В статье [2] доказана следующая

Работа выполнена при финансовой поддержке Российского фонда фундаментальных исследований, грант № 99-01-00062, и фонда INTAS, грант № 99-01080. 
Теорема А. Пусть существует положительная постоянная $C$ такая, что

$$
2^{n} \sum_{k=2^{n}}^{2^{n+1}-1}\left(\left|c_{k}-c_{k+1}\right|^{2}+\left|c_{-k}-c_{-k-1}\right|^{2}\right) \leqslant C n^{2} \quad \text { nри всех } n \geqslant 1 .
$$

Тогда условие (2) эквивалентно условию

$$
\left(\left|c_{n}\right|+\left|c_{-n}\right|\right) \ln n=o(1) \quad(\text { соответственно }=O(1)) .
$$

Из теоремы А как следствие (см. [2]) вытекает

Теорема В. Пусть существует положительная постоянная $C$ такая, что

$$
\left|c_{n}-c_{n+1}\right|+\left|c_{-n}-c_{-n-1}\right| \leqslant C \frac{\ln (n+1)}{n} \quad \text { при всех } n \geqslant 1 .
$$

Тогда условия (2) и (4) әквивалентны.

Совершенно аналогично может быть доказана

Теорема 1. Пусть для некоторого натурального $m$ существует положительная постоянная $C$ такая, что

$$
2^{n} \sum_{k=2^{n}}^{2^{n+1}-1}\left(\left|c_{k}-c_{k+m}\right|^{2}+\left|c_{-k}-c_{-k-m}\right|^{2}\right) \leqslant C n^{2} \quad \text { nри всех } n \geqslant 1 .
$$

Тогда условия (2) и (4) әквивалентны.

В частности, если для некоторого натурального $m$ и положительной постоянной С выполнено условие

$$
\left|c_{n}-c_{n+m}\right|+\left|c_{-n}-c_{-n-m}\right| \leqslant C \frac{\ln (n+1)}{n} \quad \text { при всех } n \geqslant 1,
$$

то условия (2) и (4) әквивалентны.

Теоремы А и В являются частньми случаями теоремы 1 при $m=1$. Однако теорема 1 довольно легко может быть выведена из теоремы А стандартным разделением тригонометрического ряда (1) на $m$ рядов и применением к каждому из них теоремы А.

В теоремах A, В и 1 тригонометрический ряд (1) не обязан являться рядом Фурье. Если же ряд (1) является рядом Фурье, то теорема В утверждает, что при условии (5) частные суммы ряда (1) сходятся (ограничены) в метрике $L$ тогда и только тогда, когда выполнено условие (4). Последнее утверждение улучшает результат Г. А. Фомина [3], который получается из сформулированного, если в правой части (5) взять $O(1 / n)$.

Цель этой статьи - доказать неулучшаемость условия (3) в теореме А и условия (5) в теореме В. Из неулучшаемости теорем А и В следует и неулучшаемость теоремы 1 в том же смысле.

Справедливы следующие две теоремы. 
ТЕОРема 2. Пусть последовательность положительных чисел $\{\varphi(n)\}_{n=1}^{\infty}$ такова, что величина

$$
m(\varphi ; n)=\min _{k=n, \ldots, 2 n-1} \varphi(k)
$$

удовлетворяет условию

$$
\varlimsup_{n \rightarrow \infty} 2^{n} m\left(\varphi ; 2^{n}\right) n^{-1}=\infty
$$

Тогда можно построить последовательность действительных чисел $\left\{a_{n}\right\}_{n=0}^{\infty}$ такую, что для тригонометрического ряда (1), где

$$
c_{n}=c_{-n}=\frac{a_{n}}{2} \quad \text { при всех } n \geqslant 0,
$$

будет выполнено условие

$$
\left|c_{n}-c_{n+1}\right|+\left|c_{-n}-c_{-n-1}\right| \leqslant \varphi(n) \quad \text { nри всех } n \geqslant 1
$$

и условие (4), однако

$$
\varlimsup_{n \rightarrow \infty}\left\|\sigma_{n}-S_{n}\right\|=+\infty .
$$

ТЕОРема 3. Пусть последовательность положительных чисел $\{\psi(n)\}_{n=1}^{\infty}$ такова, что

$$
\varlimsup_{n \rightarrow \infty} \psi(n) n^{-2}=\infty .
$$

Тогда мохно построить последовательность действительных чисел $\left\{a_{n}\right\}_{n=0}^{\infty}$ такую, что для тригонометрического ряда (1), определенного соотношением (7), будет выполнено условие

$$
2^{n} \sum_{k=2^{n}}^{2^{n+1}-1}\left(\left|c_{k}-c_{k+1}\right|^{2}+\left|c_{-k}-c_{-k-1}\right|^{2}\right) \leqslant \psi(n) \quad \text { nри всех } n \geqslant 1
$$

и условия (4) $и$ (9).

Отметим, что, поскольку (см. [2, лемма 3]) условие (2) всегда эквивалентно условию

$$
\left\|\widetilde{\sigma}_{n}-\widetilde{S}_{n}\right\|=o(1) \quad(\text { соответственно }=O(1)),
$$

в теоремах 2 и 3 вместо (7) можно взять соотношение

$$
c_{n}=-c_{-n}=-i \frac{a_{n}}{2} \quad \text { при всех } n \geqslant 1 .
$$

При этом теоремы 2 и 3 останутся справедливьми, просто (7) приводит к четному, а (13) к нечетному тригонометрическому ряду (1).

Отметим также, что (см. [2, лемма 3$]$ и в случае (7) и в случае (13) условие (2) эквивалентно условию (12) и эквивалентно условию

$$
\max _{m=n, \ldots, 2 n}\left\|\sum_{k=n}^{m} a_{k} e^{i k x}\right\|=o(1) \quad(\text { соответственно }=O(1)) .
$$


В частности, условие (9) эквивалентно условию

$$
\varlimsup_{n \rightarrow \infty} \max _{m=n, \ldots, 2 n}\left\|\sum_{k=n}^{m} a_{k} e^{i k x}\right\|=\infty .
$$

Теорема 3 показьвает, что в теореме А в правой части условия (3) вместо $n^{2}$ нельзя подставить никакую функцию $\psi(n)$, которая удовлетворяет условию (10). Теорема 2 утверждает, что не улучшаем порядок функции, стоящей в правой части условия (5) теоремы В. Доказательство теорем 2 и 3 и является целью этой статьи.

Прежде всего заметим, что теорема 3 легко вытекает из теоремы 2 . Действительно, пусть выполнено условие (10). Положим

$$
\varphi(n)=n^{-1} \sqrt{\psi\left(\left[\log _{2} n\right]\right)} \text { при всех } n \geqslant 2, \quad \varphi(1)=\varphi(2) .
$$

Тогда условие (6) вьполнено. По теореме 2 построим ряд (1) с условиями (8), (4) и (9). Из условия (8) видим, что левая часть (11) не больше, чем $2^{2 n} \varphi^{2}\left(2^{n}\right) \leqslant \psi(n)$. Значит, (11) вьполнено и теорема 3 доказана, если доказана теорема 2.

ДокАЗАТЕЛЬСТво ТЕОРЕмЫ 2. Пусть $\left\{\varepsilon_{k}\right\}_{k=0}^{\infty}-$ последовательность Рудина-Шапиро, т.е. $\varepsilon_{0}=1, \varepsilon_{2 k}=\varepsilon_{k}, \varepsilon_{2 k+1}=(-1)^{k} \varepsilon_{k}$ при $k \geqslant 0$. Пусть далее квадратные скобки обозначают целую часть числа. При любых натуральных $n, m$, где $n \geqslant 3 m-1$, положим

$$
\begin{aligned}
& U(n, m ; x)=\sum_{k=[n /(2 m-1)]+1}^{[2 n /(2 m-1)]-1} \varepsilon_{k} e^{i k x}, \\
& T(n, m ; x)=\frac{1}{\sqrt{n m}}\left(\sum_{j=0}^{m-1} e^{i j x}\right)^{2} U(n, m ;(2 m-1) x) .
\end{aligned}
$$

Тогда из (15) и (16) имеем

$$
\begin{aligned}
T(n, m ; x) & =\sum_{\nu=n+1}^{2 n-1} d_{\nu}(n, m) e^{i \nu x} \\
|T(n, m ; x)| & =\frac{1}{\sqrt{n m}}\left|\frac{\sin (m x / 2)}{\sin (x / 2)}\right|^{2}|U(n, m ;(2 m-1) x)| .
\end{aligned}
$$

Далее через $C_{1}, C_{2}, \ldots$ обозначается абсолютная положительная постоянная, в каждом случае своя. По известному свойству полиномов Рудина-Шапиро (см. [4], [5], [6, гл. 4, теорема 1]) верна оценка

$$
C_{1} \sqrt{n} \leqslant \sqrt{m}\|U(n, m)\| \leqslant C_{2} \sqrt{n} \quad \text { при всех } n \geqslant 3 m-1 .
$$

Из $(18)$ и $(19)$, полагая $I(j)=[\pi(2 j-1) /(2 m-1), \pi(2 j+1) /(2 m-1)]$, получаем

$$
\begin{aligned}
\sqrt{n m}\|T(n, m)\| \leqslant & \frac{1}{2 \pi} \int_{x \in I(0)} m^{2}|U(n, m ;(2 m-1) x)| d x \\
& +\sum_{j=1}^{m-1} \frac{1}{2 \pi} \int_{|x| \in I(j)} \frac{(2 m-1)^{2}}{(2 j-1)^{2}}|U(n, m ;(2 m-1) x)| d x \\
= & \frac{m^{2}}{(2 m-1)}\|U(n, m)\|+\sum_{j=1}^{m-1} \frac{(2 m-1)}{(2 j-1)^{2}}\|U(n, m)\| \leqslant C_{3} \sqrt{n m} .
\end{aligned}
$$


Следовательно, всегда $\|T(n, m)\| \leqslant C_{3}$. С другой стороны, при $n \geqslant 3 m-1$ имеем

$$
\begin{aligned}
\sqrt{n m}\|T(n, m)\| & \geqslant \frac{1}{2 \pi} \int_{x \in I(0)} \frac{(2 m)^{2}}{\pi^{2}}|U(n, m ;(2 m-1) x)| d x \\
& =\frac{(2 m)^{2}}{\pi^{2}(2 m-1)}\|U(n, m)\| \geqslant C_{4} \sqrt{n m} .
\end{aligned}
$$

Поэтому

$$
C_{4} \leqslant\|T(n, m)\| \leqslant C_{3} \quad \text { при всех } n \geqslant 3 m-1 .
$$

Поскольку

$$
\left(\sum_{j=0}^{m-1} e^{i j x}\right)^{2}=\sum_{s=0}^{m-1}(s+1) e^{i s x}+\sum_{s=m}^{2 m-2}(2 m-1-s) e^{i s x},
$$

то из (16) и (17) выводим, что

$$
d_{k(2 m-1)+s}(n, m)=\frac{\varepsilon_{k} \min (s+1,2 m-1-s)}{\sqrt{n m}}
$$

при $s=0, \ldots, 2 m-2, k=[n /(2 m-1)]+1, \ldots,[2 n /(2 m-1)]-1$ и

$$
d_{\nu}(n, m)=0
$$

при $\nu<(2 m-1)([n /(2 m-1)]+1)$ и $\nu>(2 m-1)([2 n /(2 m-1)]-1)+2 m-2$. Поэтому

$$
\begin{gathered}
\max _{\nu}\left|d_{\nu}(n, m)\right|=\frac{\sqrt{m}}{\sqrt{n}} \quad \text { при всех } n \geqslant 3 m-1, \\
\max _{\nu}\left|d_{\nu}(n, m)-d_{\nu+1}(n, m)\right| \leqslant \frac{2}{\sqrt{n m}} .
\end{gathered}
$$

Возьмем теперь любую стремящуюся к бесконечности последовательность положительных чисел $\left\{\gamma_{k}\right\}_{k=1}^{\infty}$, например $\gamma_{k}=k$. Построим строго возрастающую последовательность натуральных чисел $\left\{n_{k}\right\}_{k=1}^{\infty}$ так, что при всех натуральных $k$ выполнены следуюшие условия: $n_{k}$ - степень двойки, $\log _{2} n_{k} \geqslant 2 \gamma_{k}^{-2}, \sqrt{n_{k}} \geqslant \gamma_{k}^{2} \log _{2} n_{k}$, $n_{k} m\left(\varphi ; n_{k}\right) \geqslant 4 \gamma_{k}^{3} \log _{2} n_{k}$. Такое построение возможно в силу условия (6). Пусть

$$
m_{k}=\left[n_{k}\left(\gamma_{k}^{2} \log _{2} n_{k}\right)^{-2}\right] \quad \text { при } k \geqslant 1 .
$$

Тогда натуральные $m_{k}$ удовлетворяют условию: $n_{k} \geqslant 3 m_{k}$ при всех $k \geqslant 1$. Последовательность действительных чисел $\left\{a_{n}\right\}_{n=1}^{\infty}$ определим из формального равенства

$$
\sum_{k=1}^{\infty} \gamma_{k} T\left(n_{k}, m_{k} ; x\right)=\frac{1}{2} a_{0}+\sum_{n=1}^{\infty} a_{n} e^{i n x} .
$$

Тогда в силу (20)

$$
\left\|\sum_{n=n_{k}}^{2 n_{k}-1} a_{n} e^{i n x}\right\|=\left\|\gamma_{k} T\left(n_{k}, m_{k}\right)\right\| \geqslant C_{4} \gamma_{k} \rightarrow \infty \quad \text { при } k \rightarrow \infty
$$


и условие (14), а значит и (9), выполнено. Из (17) и (21) при $n \in\left[n_{k}, n_{k+1}\right)$ имеем $\left|a_{n}\right| \log _{2} n=0$, если $n \geqslant 2 n_{k}$, и $\left|a_{n}\right| \log _{2} n \leqslant \gamma_{k} \sqrt{m_{k} / n_{k}} \log _{2}\left(2 n_{k}\right) \leqslant \gamma_{k}^{-1}\left(1+1 / \log _{2} n_{k}\right)$ при $n<2 n_{k}$. Поэтому $a_{n} \ln n \rightarrow 0$ при $n \rightarrow \infty$ и условие (4) вьполнено. Наконец, при $n \in\left[n_{k}, n_{k+1}\right)$ в силу (22) и (17) верна оценка

$$
\left|a_{n}-a_{n+1}\right| \leqslant 2 \gamma_{k}\left(n_{k} m_{k}\right)^{-1 / 2} \leqslant \frac{2 \sqrt{2} \gamma_{k}^{3} \log _{2} n_{k}}{n_{k}} \leqslant m\left(\varphi ; n_{k}\right) \leqslant \varphi(n),
$$

если $n<2 n_{k}$, и $\left|a_{n}-a_{n+1}\right|=0$, если $n \geqslant 2 n_{k}$. Следовательно, условие (8) также выполнено. Теорема 2 полностью доказана.

\section{СПИСОК ЦИТИРОВАННОЙ ЛИТЕРАТУРЫ}

[1] Бари Н. К. Тригонометрические ряды. М.: Физматгиз, 1961.

[2] Белов А. С. // Изв. Тульского гос. ун-та. Сер. Матем. Мех. Информатика. 1998. Т. 4. № 1. C. $40-46$.

[3] Фомин Г. А. Über die Konvergenz im Mittel von Fourierreihen // Матем. сб. 1979. Т. 110. №2. C. 251-265.

[4] Rudin W. Some theorems on Fourier coefficients // Proc. Amer. Math. Soc. 1959. V. 10. № 4. P. 855-859.

[5] Brillhart J., Carlitz L. Note on the Shapiro polynomials // Proc. Amer. Math. Soc. 1970. V. 25. № 1. P. 114-118.

[6] Кашин Б. С., Саакян А. А. Ортогональные ряды. М.: Наука, 1984.

Ивановский государственный университет 